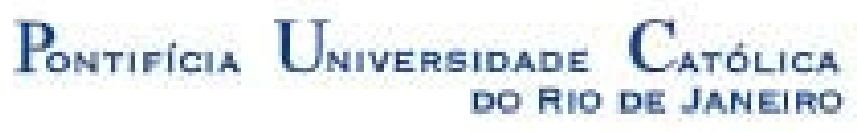

Paulo Roberto de Oliveira Bastos Junior

\title{
Elicitação de requisitos de software através da utilização de questionários
}

\author{
Dissertação de Mestrado
}

Dissertação apresentada ao Programa de Pós-graduação em Informática da PUC-Rio como requisito parcial para obtenção do título de Mestre em Informática.

Orientador: Julio César Sampaio do Prado Leite

Rio de Janeiro Abril de 2005 
Paulo Roberto de Oliveira Bastos Junior

\title{
Elicitação de requisitos de software através da utilização de questinários
}

\begin{abstract}
Dissertação apresentada como requisito parcial para a obtenção do grau de Mestre pelo Programa de Pósgraduação em Informática do Departamento de Informática do Centro Técnico e Científico da PUCRio. Aprovada pela Comissão Examinadora abaixo assinada.
\end{abstract}

\section{Prof. Julio Cesar Sampaio do Prado Leite \\ Orientador \\ Departamento de Informática - PUC-Rio}

Prof. Carlos José Pereira de Lucena

Departamento de Informática - PUC-Rio

Prof. Arndt von Staa

Departamento de Informática - PUC-Rio

Prof. Karin Koogan Breitman

Departamento de Informática - PUC-Rio

Prof. José Eugenio Leal

Coordenador Setorial do Centro

Técnico Científico - PUC-Rio

Rio de janeiro, 26 de abril de 2005 
Todos os direitos reservados. É proibida a reprodução total ou parcial do trabalho sem autorização do autor, do orientador e da universidade.

\section{Paulo Roberto de Oliveira Bastos Junior}

Graduou-se em Engenharia de Computação pela PUC-Rio em 2001. Área de interesse acadêmico: Engenharia de software, mais especificamente a sub-área de Engenharia de Requisitos. Atualmente trabalhando na multinacional de consultoria Accenture como Consultor, atuando como coordenador da equipe de operações do sistema B2B Canal Cliente da Petrobras, realizando as seguintes atividades: levantamento de requisitos com o cliente; análise e especificação (técnica e funcional) das atividades a serem desenvolvidas; participação nas reuniões/Atas e definições do projeto.

Ficha catalográfica

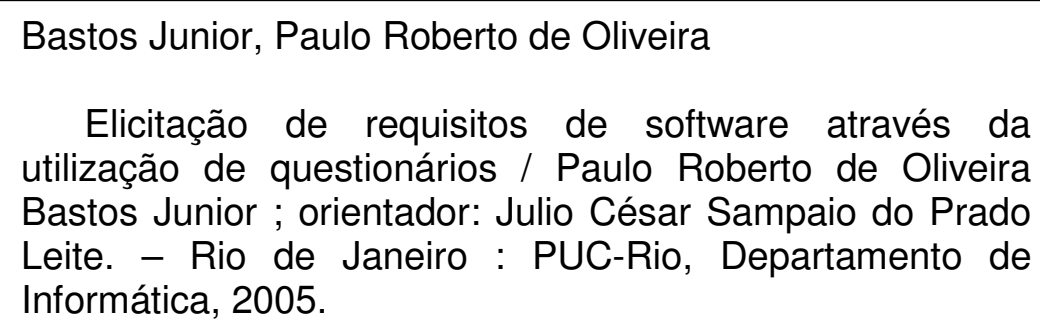
utilização de questionários / Paulo Roberto de Oliveira Bastos Junior ; orientador: Julio César Sampaio do Prado Leite. - Rio de Janeiro : PUC-Rio, Departamento de Informática, 2005.

$$
88 \mathrm{f.} ; 30 \mathrm{~cm}
$$

Dissertação (mestrado) - Pontifícia Universidade Católica do Rio de Janeiro, Departamento de Informática.

Inclui bibliografia

1. Informática - Teses. 2. Engenharia de requisitos. 3. Elicitação de requisitos. 4. Questionários. I. Leite, Julio César Sampaio do Prado. II. Pontifícia Universidade Católica do Rio de Janeiro. Departamento de Informática . III. Título. 


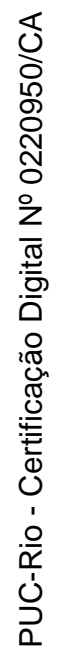

A meus pais 


\section{Agradecimentos}

A meus pais, pela força e incentivo.

A Deus, por me iluminar e me dar forças para atingir esse objetivo.

A PUC-Rio, pelos auxílios concedidos, sem os quais esse trabalho não poderia ser realizado.

A Julio César Sampaio do Prado Leite, orientador da dissertação, pelo apoio dado durante essa jornada. 


\section{RESUMO}

Bastos Junior, Paulo Roberto de Oliveira; Leite, Julio César Sampaio do Prado; Elicitação de requisitos de software através da utilização de questionários. Rio de Janeiro, 2005. 88p. Dissertação de Mestrado Departamento de Informática, Pontifícia Universidade Católica do Rio de Janeiro.

Um dos possíveis meios utilizados para a coleta de fatos na elicitação de requisitos é o uso do questionário. Um questionário consiste num documento usado para guiar uma ou mais pessoas a responder a uma ou mais perguntas. A elaboração de um questionário é um processo bem mais complexo do que possa aparentar. Um questionário mal formulado pode levar a considerações erradas, o que acaba sendo prejudicial ao projeto em questão. Não existe um método padrão para a construção de questionários, porém existem recomendações de diversos autores com relação a essa importante tarefa no processo de pesquisa científica. $\mathrm{O}$ trabalho aqui apresentado detalha a técnica de questionários, identificando as etapas necessárias e comuns à criação de um questionário eficaz. Será proposto um método para a construção de perguntas de questionários, que utiliza como base uma listagem de requisitos para a construção de questionários de qualidade, obtida após a realização de uma extensa pesquisa nas áreas de ciências sociais e marketing. Posteriormente apresentamos uma ferramenta utilizada para elicitação de requisitos de software, através da utilização de questionário, questionário esse gerado através do método proposto no presente trabalho.

\section{Palavras-Chave}

Engenharia de Requisitos; elicitação de requisitos; informática; questionários. 


\section{ABSTRACT}

Bastos Junior, Paulo Roberto de Oliveira; Leite, Julio César Sampaio do Prado; Software requirements elicitation through the use of questionnaires. Rio de Janeiro, 2005. 88p. Master degree thesis - Computer Science Department, Pontifícia Universidade Católica do Rio de Janeiro.

Questionnaire is one of the techniques available for requirements elicitation. A questionnaire is a document used to guide one or more people to answer one or more questions. The elaboration of a questionnaire is a process more complex than it can make look like. A questionnaire that is not well formulated can lead to unreliable information and may be harmful to the project in question. Although there is no standard method for the construction of questionnaires, there are recommendations from diverse authors with regard to this important task in the process of eliciting information. The work presented here details a technique for identifying the necessary and common stages for the creation of a questionnaire. A method for the construction of questions is proposed, which uses as base a list of requirements for the construction of quality questionnaires, obtained from the literature in the areas of social sciences and marketing. A tool for the elicitation of software requirements, by means of questionnaires is presented.

\section{Keywords}

Software Engineering; requirements elicitation; questionnaires. 


\section{SUMÁRIO}

1. Introdução 10

1.1. Motivação 10

1.2. Objetivo da dissertação 12

1.3. Organização da dissertação 13

2. Revisão da literatura 14

2.1. A utilização de questionários na Engenharia de Requisitos 14

2.2. A técnica GQM 27

3. Questionários 30

3.1. Tipos de questões 30

3.2. Requisitos para a construção de questionários de boa $\begin{array}{ll}\text { qualidade } & 37\end{array}$

3.3. Erros comumente cometidos num processo de aplicação de questionários 41

3.4. Avaliação do questionário $\quad 42$

4. Um processo para a elaboração de perguntas de questionários para a elicitação de requisitos de software $\quad 45$

4.1. Definição do processo 45

5. Experimento prático 62

5.1. Características gerais da ferramenta 62

5.2. Apresentação da ferramenta 66

5.3. Método utilizado para análise das respostas e priorização dos requisitos 
6. Avaliação, contribuição e desdobramentos 79

6.1. Avaliação do método e da ferramenta propostos 79

6.2. Comparação com outras iniciativas 80

6.3. Contribuições 83

6.4. Trabalhos futuros $\quad 84$

7. Referências bibliográficas 85 